\section{Surgical outcome of safe surgery system trabeculectomy combined with cataract extraction}

RR Khandelwal' ${ }^{1}$ D Raje ${ }^{2}$, A Rathi ${ }^{1}$, A Agashe ${ }^{1}$, M Majumdar ${ }^{1}$ and $\mathrm{R}$ Khandelwal ${ }^{1}$

\begin{abstract}
Purpose To determine the efficacy of safe surgery system trabeculectomy combined with manual small incision cataract surgery/ phacoemulsification in primary glaucoma coexistent with cataract.

Methods This is a retrospective analysis of 105 cases who underwent single-site combined surgery between January 2008 and December 2009. Safe surgery system trabeculectomy with diffuse and posterior application of mitomycin $\mathrm{C}$ was performed in all cases. Cataract extraction was done either by Manual Small Incision Cataract Surgery (MSICS) or phacoemulsification. Main outcome measures were success rate of trabeculectomy, as determined by four different IOP goals and incidence of postoperative complications. Analysis was performed using R-2.15, and the significance was tested at $5 \%$ level.

Results The minimum follow-up period was 12 months. The overall success rates (with or without medication) when safe surgery system trabeculectomy was combined with MSICS were 91,70 , and $51 \%$ for IOP $\leq 18$, $\leq 15$, and $\leq 12 \mathrm{~mm} \mathrm{Hg}$, respectively, and target IOP was achieved in $72 \%$ cases. The mean IOP reduction was $43.8 \%$ with MSICS and $42.08 \%$ with phacoemulsification. The surgical outcome was not significantly different for both techniques. Postoperative complications were infrequent and comparable.

Conclusion The Safe Surgery System

Trabeculectomy combined with cataract surgery offers excellent IOP control with minimal postoperative complications. It offers an effective and improved solution for primary glaucoma coexistent with cataract found in developing countries.
\end{abstract}

Eye (2015) 29, 363-370; doi:10.1038/eye.2014.294; published online 12 December 2014

\title{
Introduction
}

Glaucoma is the second leading cause of blindness in India, which is predicted to affect approximately $20 \%$ of the world's glaucoma population by $2020 .^{1}$ The prevalence of glaucoma in India has been reported by various epidemiological studies and is estimated to be approximately 11.2 million people aged $\geq 40$ years. ${ }^{2}$ In developing countries, glaucoma is generally diagnosed at an advanced stage, only when the patient seeks advice for cataract surgery. Trabeculectomy is thus performed as a primary procedure with cataract extraction and implantation of an intraocular lens (IOL). 3,4 Combined surgery has an advantage of controlling IOP without requiring the use of lifelong anti-glaucoma medication.

Although primary trabeculectomy is frequently combined with manual small incision cataract surgery (MSICS) and extracapsular cataract surgery (ECCE), a few data are available on the surgical outcome of combined surgery from developing countries. ${ }^{3,5-7}$ Combined surgery is effective in lowering IOP but carries risk of postoperative complications and failure. ${ }^{8-10}$ The incidence of complications can be reduced by adopting the new surgical technique of safe surgery system trabeculectomy developed by Peng Khaw at Moorfields Eye Hospital. ${ }^{11,12}$ It is an evidencebased, step-by-step approach to trabeculectomy surgery that preserves vision in patients with glaucoma by minimizing complications while maintaining a desired IOP. ${ }^{12,13}$ This technique helps to reduce the number of major complications of trabeculectomy, such as hypotony, cystic blebs, and endophthalmitis, thereby improving the overall surgical outcome. The procedure is simple, requires minimal equipment, and can be easily mastered. ${ }^{14}$
${ }^{1}$ Department of Ophthalmology, NKP Salve Institute of Medical Sciences and Lata Mangeshkar Hospital, Nagpur, India

${ }^{2}$ MDS Bioanalytics, Nagpur, India

Correspondence: RR Khandelwal, Department of Ophthalmology, NKP Salve Institute of Medical Sciences and Lata Mangeshkar Hospital, Digdoh Hills, Hingna Road, Nagpur 440019, India Tel: +91 7104 306100; Fax: +91 7104306111. E-mail: rekha.khandelwal@ gmail.com

Received: 20 December 2013

Accepted in revised form: 30 September 2014 Published online: 12 December 2014 
The long-term results of safe surgery system trabeculectomy published earlier showed excellent IOP control with few complications. ${ }^{15,16}$ Hence, this study was designed to evaluate and compare the results of safe surgery system as a combined procedure with MSICS or phacoemulsification in advanced primary glaucoma coexistent with cataract.

\section{Material and methods}

In this non-randomized comparative study, the case records of 105 patients (retrieved from the computerized database) who underwent safe surgery system trabeculectomy with cataract extraction (MSICS or phacoemulsification) between January 2008 and December 2009 at a rural-based hospital of Central India were retrieved after approval by the institutional review board. All cases of primary open angle glaucoma (POAG) and primary angle closure glaucoma (PACG) with visually significant cataract in otherwise healthy eye were included irrespective of their preoperative best-corrected visual acuity (BCVA). Intraocular pressure (IOP) $>21 \mathrm{~mm} \mathrm{Hg}$ (Goldmann applanation tonometer by Inami and Co., Tokyo, Japan) with typical glaucomatous optic neuropathy (cup: disc ratio $\geq 0.7$ or asymmetry of $\geq 0.2$ ) in one or both eyes and/or visual field loss on 24-2 Humphrey Field Analyzer (Carl-Zeiss Meditec, Inc, Dublin, CA, USA) were the selection criteria for primary trabeculectomy with cataract extraction. For patients unable to perform reliable field testing, severe glaucomatous disc damage (cup: disc ratio $\geq 0.8$ ) with $\mathrm{IOP}>21 \mathrm{~mm} \mathrm{Hg}$ were also operated upon. The choice of cataract surgery was based on the economical status of patients and grading of nuclear sclerosis. Patients of secondary glaucoma (lens induced, pseudoexfoliation, pigmentary, neovascular, uveitic, steroid induced, angle recession glaucoma, previous failed trabeculectomy) and patients with $<12$ months of follow-up were excluded from the study.

The preoperative data included age, sex, ocular history, preexisting medical condition, type of glaucoma, visual acuity using Snellen's distance acuity chart, anterior segment examination, applanation IOP, stereoscopic assessment of the disc by slit lamp indirect ophthalmoscopy, gonioscopy, A-scan biometry for IOL power calculations, number of glaucoma medications, and preset target IOP. ${ }^{17}$ Visual field examination using a Humphrey Field Analyzer was attempted whenever possible.

\section{Statement of ethics}

We certify that all applicable institutional and governmental regulations concerning the ethical use of human volunteers were followed during this research.

\section{Surgical technique}

The safe surgery system trabeculectomy was based on the previously described technique with modifications for combining it with cataract extraction as described below. ${ }^{11-14}$

Under peribulbar anesthesia (2\% Xylocaine, $2 \%$ Bupivacaine, Hyaluronidase $37.5 \mathrm{IU} / \mathrm{ml}$ ), the periocular area was cleaned with 5\% Povidone iodine for $3 \mathrm{~min}$. A superior rectus traction suture was taken with 6-0 silk, and a fornix-based conjunctival flap was dissected superiorly; Tenon's capsule was separated. The conjunctiva was dissected backwards for $8-10 \mathrm{~mm}$ for the wide application of antimetabolite sponges. Three mitomycin C $(0.2 \mathrm{mg} / \mathrm{ml})$ soaked sponges were folded and applied below the conjunctiva, keeping the edges at a distance. After $2 \mathrm{~min}$, the sponges were removed, and the area was thoroughly irrigated with $20 \mathrm{ml}$ Ringer's lactate.

For MSICS, a partial thickness scleral tunnel (flap) of $5.5-7 \mathrm{~mm}$ was made $3 \mathrm{~mm}$ posterior to the limbus with a crescent blade. The dissection was performed $1-2 \mathrm{~mm}$ into the clear cornea, and scleral pockets were fashioned in the same plane, leaving the sides of the scleral tunnel intact. A corneal paracentesis was made temporally, through which a capsulorhexis $(5-6 \mathrm{~mm})$ was performed after injecting visco-elastics. The anterior chamber was entered with a 3.2-mm keratome at 12 o'clock and the inner lip of tunnel was enlarged to approximately 8-9 mm. After hydrodissection, the nucleus was rotated and prolapsed into the anterior chamber using a sinsky hook. The nucleus was delivered with the help of a wire vectis and a sinsky hook using the sandwich technique after injecting visco-elastic (Viscomet-PF, Milmet (Sun Pharmaceuticals Industries Ltd), Mumbai, India) both above and below the nucleus. The remaining cortex was aspirated using a simcoe two-way irrigation-aspiration cannula. A polymethyl methacrylate (PMMA) IOL (single piece, optic diameter 5.5 or $6 \mathrm{~mm}$, modified C loop with $10^{\circ}$ angulation, S3602; Aurolab, Madurai, India) was placed in the bag and rotated to a horizontal position as shown in Figure 1.

For phacoemulsification, a $3.5-\mathrm{mm}$ partial thickness scleral tunnel, $2.5 \mathrm{~mm}$ posterior to the limbus with side incision at $1 \mathrm{~mm}$ from cornea, was fashioned into a clear cornea using a crescent knife. A paracentesis was performed, and the anterior chamber was entered using a 2.8-mm keratome blade. Phacoemulsification was performed using the stop and chop technique. After cortical aspiration, the wound was enlarged with a crescent knife. A hydrophilic foldable lens (poly(hydroxyl ethyl methacrylate), with a dual haptic $6 \mathrm{~mm}$ optic diameter, Auroflex-FH5600SQ, Aurolab) was 

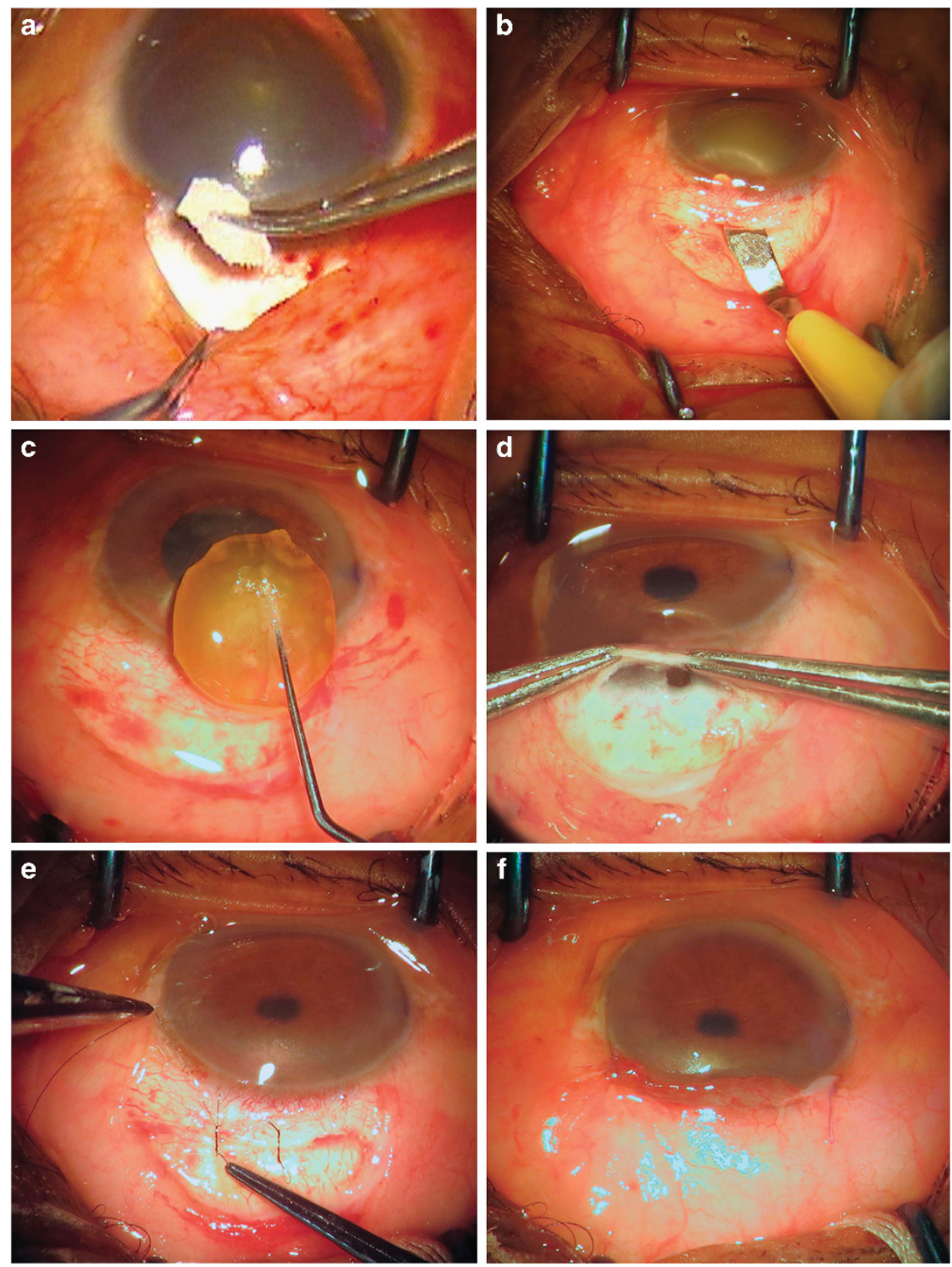

Figure 1 Intraoperative photograph showing surgical procedure of MSICS-trabeculectomy. (a) Fornix-based conjunctival flap with diffuse and posterior application of MMC $(0.2 \mathrm{mg} / \mathrm{ml}$, for $2 \mathrm{~min})$, without touching the conjunctival edges. (b) Partial thickness scleral tunnel, $3 \mathrm{~mm}$ posterior to the limbus, and $1-2 \mathrm{~mm}$ into the clear cornea with scleral pockets on the sides. (c) The nucleus delivered by the 'sandwich technique'. (d) Trabeculectomy performed by excising a block of $0.5-\mathrm{mm}$ tissue from the posterior lip of the scleral tunnel using a Kelly's Descemet's membrane punch. (e) The scleral flap closed with adjustable 10-0 nylon sutures for minimal spontaneous filtration. (f) The conjunctival incision closed using 8-0 vicryl sutures in a watertight manner.

inserted into the bag with the use of a disposable cartridge and injector.

After the cataract extraction (MSICS/phacoemulsification) and implantation of PCIOL, visco-elastic was aspirated and acetylcholine was injected to constrict the pupil. The trabeculectomy was performed by excising a block of $0.5-\mathrm{mm}$ tissue from the posterior lip of the scleral tunnel using a Kelly's descemet's membrane punch, followed by iridectomy. Two adjustable 10-0 monofilament nylon sutures (with four throws) were taken on either side of the punch area to close the scleral tunnel as described in safe surgery system trabeculectomy. Additional interrupted sutures were taken at the corners of the scleral tunnel when it was enlarged for a large brown and hard nuclear cataract. Conjunctival closure was achieved by 10-0 nylon using anchoring corneal suture technique to seal the conjunctiva in a watertight manner under tension. The patency of trabeculectomy was tested at the end of the surgery, by injecting Ringer's lactate through the side port and observing formation of diffuse conjunctival bleb without any leak. A subconjunctival injection of corticosteroids (Dexamethasone) and 
antibiotics (Gentamycin sulphate) was given in the inferior fornix.

Postoperatively, the patients were treated with ciprofloxacin $0.3 \%$ four times daily for 2 weeks and prednisolone acetate $1 \%$ drops six times per day for 1 week; the dosage was then gradually tapered over 2 months. The supplemental use of antiglaucoma medications and cycloplegics was based on the IOP and the level of intraocular inflammation. Patients were examined as per the conventional postoperative protocol followed for combined surgery in our set up. They were evaluated on postoperative day 1 , day 3 , first week, at 1 month, 3 months, and every 3 months thereafter with additional visits as and when required. The postoperative IOP (by Goldman applanation tonometer), BCVA on Snellen's distant acuity chart, and complications were recorded on each visit.

\section{Outcome measures}

Success was measured on the basis of four different IOP goals (IOP $\leq 18, \leq 15, \leq 12 \mathrm{~mm} \mathrm{Hg}$, achieving a preset target IOP, 17,18 and mean IOP reduction from baseline). A complete success (CS) was defined as IOP control without medication, whereas with medication was treated as qualified success (QS). Overall success was defined as IOP control with or without medications. Sustained IOP $<5 \mathrm{~mm} \mathrm{Hg}$ on two visits with anatomic changes or no PL vision postoperatively was considered a failure. A bleb was defined as cystic if it was described as cystic in medical case record forms (by text) or demarcated (by diagram) and noted as avascular on two consecutive visits. An ideal bleb was defined as diffuse and mildly elevated with normal vascularity. Seidelpositive blebs within the first month of follow-up were classified as wound leaks and those occurring after 1 month were labeled as bleb leaks. Bleb-related infection included bleb-related endophthalmitis and blebitis (discharging eye with opacification of bleb). Visual outcome was defined as the presence or absence of improvement in visual acuity as compared with the preoperative acuity of vision on Snellen's chart.

\section{Statistical analysis}

The summery statistics for the baseline characteristics of the patients were obtained and compared using the appropriate tests, such as the $t$-test for the independent samples and Chi-squared test. The association of success (complete/qualified) with surgery type was evaluated using a Chi-squared test. Furthermore, to compare the success rates by surgery type, Kaplan-Meier (KM) analysis was performed, and a log-rank test was used to evaluate the statistical significance of difference in the survival plots representing the two groups. Preoperative and postoperative mean IOP levels were compared using paired $t$-test with Bonferroni multiple testing correction in each group. The analyses were performed using R-2.15 (R Core Team 2012, Foundation for Statistical Computing, Vienna, Austria), and the significance was tested at the $5 \%$ level.

\section{Results}

Out of the 105 combined surgeries performed (by a single surgeon) during the study period, safe surgery system trabeculectomy was combined with 72 MSICS (MSICStrab) and 33 phacoemulsification (phaco-trab) cases. As per the selection criteria, 53 MSICS-trab and 22 phacotrab were included in the analysis. There were 25 POAG and 50 PACG patients in whom first operated eye was considered for analysis. The mean follow-up was 13.73 months (range 12-18 months). The baseline characteristics of patients are shown in Table 1.

\section{Main outcome measures}

Of all the combined cases, surgical success of safe surgery system trabeculectomy was achieved in 69 (92\%) out of the 75 cases, and preset target IOP (without or with medication) was achieved in $58(77.3 \%)$ cases.

The overall success was achieved in 48 (90.6\%), 37 (69.8\%), and $27(50.9 \%)$ MSICS-trab cases and 21 (95.4\%), $16(72.7 \%)$, and $20(90.9 \%)$ phaco-trab cases for IOP $\leq 18$, $\leq 15$, and $\leq 12 \mathrm{~mm} \mathrm{Hg}$, respectively.

The target IOP achieved was 38 (71.7\%) in MSICS-trab, and it was 20 (90.9\%) in phaco-trab cases. The success with or without medication was insignificantly associated between the surgery types $(P>0.05)$. The success of surgery is shown in Table 2.

The difference between the KM plots for safe surgery system trabeculectomy combined with MSICS or phacoemulsification was not statistically significant as per the log-rank test $(P>0.05)$. KM plots characterizing the success rates are shown in Figure 2.

At the last follow-up, a significant reduction in the mean IOP from baseline was observed in all the combined cases from postoperative day 1, irrespective of the type of cataract surgery. The mean IOP was reduced from 26.5 (SD 5) $\mathrm{mm} \mathrm{Hg}$ to 14.9 (SD 3) $\mathrm{mm} \mathrm{Hg}$ in the MSICS-trab cases while from 25.2 (SD 5) $\mathrm{mm} \mathrm{Hg}$ to 14.6 (SD 3 ) $\mathrm{mm} \mathrm{Hg}$ in the phaco-trab cases.

The visual acuity improvement was noted in 70 cases (93.3\%). There were $\geq 2$ lines of improvement on Snellen's acuity in $26(49.1 \%)$ of the MSICS-trab and in $12(54.5 \%)$ of the phaco-trab cases. The vision improvement achieved was not statistically different in the two types of combined cases $(P>0.05)$. 
Table 1 Baseline characteristics

\begin{tabular}{|c|c|c|c|}
\hline \multirow[t]{2}{*}{ Characteristics } & \multicolumn{2}{|c|}{ Surgery type } & \multirow[t]{2}{*}{ P-value } \\
\hline & MSICS-trab & Phaco-trab & \\
\hline Number of cases & $53(70.6 \%)$ & $22(29.4 \%)$ & \\
\hline Mean age in years (SD) & $61.1(8.76)$ & $60.36(7.05)$ & $0.6948^{\mathrm{a}}$ \\
\hline $\operatorname{Sex}(M / F)$ & $24 / 29$ & $13 / 9$ & $0.4035^{\mathrm{b}}$ \\
\hline \multicolumn{4}{|l|}{ Glaucoma type } \\
\hline POAG & 16 & 9 & $0.5302^{c}$ \\
\hline PACG & 37 & 13 & \\
\hline \multicolumn{4}{|l|}{ IOP before surgery $(\mathrm{mm} \mathrm{Hg})$} \\
\hline Mean (SD) & $26.5(5)$ & $25.2(5)$ & $0.2876^{\mathrm{a}}$ \\
\hline \multicolumn{4}{|l|}{$\begin{array}{l}\text { Pre-op antiglaucoma } \\
\text { medications }\end{array}$} \\
\hline Mean (SD) (Range 0-4) & $1.8(0.7)$ & $1.7(0.6)$ & $0.6109^{d}$ \\
\hline $\begin{array}{l}\text { Minimum follow-up } \\
\text { (months) }\end{array}$ & 12 & 12 & \\
\hline
\end{tabular}

Intraoperative complications during the combined surgery were not observed for any patient. Postoperative complications were uncommon as shown in Table 3.

\section{Discussion}

To the best of our knowledge, this is the first study on the surgical outcome of combined procedure using safe surgery system trabeculectomy with cataract extraction at 12 months follow-up. ${ }^{11-14}$ An evidence-based study on combined procedures focused on the important issues in the management of glaucoma coexistent with cataract. ${ }^{9}$ There was insufficient evidence in favor of alternative filtering surgeries superior to trabeculectomy or twostage procedure being better than one-stage combined surgery. There was also weak evidence for phacoemulsification lowering more IOP than nucleus expression (ECCE/MSICS) practiced in developing countries. Phacoemulsification with foldable lens has the advantage of smaller incision and earlier visual rehabilitation, but for the developing countries, the increased cost is a major hurdle. Hence, single-site safe surgery trabeculectomy was combined with MSICS/ phacoemulsification in the present study. The direct head-to-head comparison between studies is difficult due to different definitions of success, different antimetabolite use, and varying follow-up period. There was a double number of PACG $(n=50)$ as compared with POAG $(n=25)$ cases in our study. The advanced glaucoma due to PACG remains asymptomatic (chronic angle closure) in many Asian countries and are detected accidently at the time of cataract surgery. Combining cataract surgery with trabeculectomy has additional pressure benefit in such patients.

The mean IOP reduction was $>40 \%$ from the baseline IOP (43.76\%) in MSICS-trab and $42.08 \%$ in phaco-trab cases in our series of combined cases, which was better than combined surgery results reported earlier. ${ }^{3,6}$ Stark et $a l^{19}$ found mean IOP reduction of $19.2 \%$ in a retrospective analysis of phacotrabeculectomy without antimetabolites. It is likely that greater percentage reduction in IOP were achieved owing to higher preoperative IOP levels, which must be kept in mind while comparing different studies. There was no statistically significant difference in IOP reduction between the two combined surgery groups $(P<0.001)$. Our MSICS-trab results were comparable to a recently published retrospective study on combined surgery performed through a scleral tunnel from South India, which had 6 months follow-up. ${ }^{7}$

The overall success achieved (IOP $\leq 18 \mathrm{~mm} \mathrm{Hg}$ ) without or with medication was $>90 \%$ when combined with either MSICS $(90.6 \%)$ or phacoemulsification (95.4\%), which was better than combined surgery results on Indian eyes. ${ }^{3,19}$ We also analyzed the success taking IOP $\leq 15$ and $\leq 12 \mathrm{~mm} \mathrm{Hg}$ in both types of surgeries. The success reported was 70 and $51 \%$ with MSICS and 73 and $50 \%$ with phacoemulsification, respectively. A success rate of $58 \%$ (IOP $\leq 15 \mathrm{~mm} \mathrm{Hg}$ at 6 months) was reported by a retrospective study on 5-flurouracil-augmented combined surgery from East Africa. ${ }^{4}$ A retrospective study analyzed 56 eyes that underwent safe surgery trabeculectomy and reported a success rate of 90.9 and $61.4 \%$ for $\mathrm{IOP} \leq 18$ and $\leq 14 \mathrm{~mm} \mathrm{Hg}$, respectively, beyond 12 months follow-up. ${ }^{15}$ Another prospective study of safe surgery system trabeculectomy on 39 eyes showed that $74 \%$ had an IOP $\leq 15 \mathrm{~mm} \mathrm{Hg}$ for $>90 \%$ of their follow-up period of 3 years, without any antiglaucoma medications. Our results of combined surgery were comparable with the studies published on medium- and long-term success of safe surgery system trabeculectomy (without cataract extraction) published earlier. ${ }^{15,16}$

A complete success (IOP $\leq 18 \mathrm{~mm} \mathrm{Hg}$, without medication) was observed in $62.3 \%$ of MSICS-trab and $50 \%$ in phaco-trab cases. We analyzed preset target IOP achieved in combined cases, which was 71.7 and $90.9 \%$ in the MSICS-trab and the phaco-trab, respectively. ${ }^{17}$

Postoperative improvement in visual acuity was found in $>90 \%$ of all combined cases. Vision improvement was found in $92.45 \%$ in MSICS-trab and $95.5 \%$ in phaco-trab cases. There was no significant difference found in the two groups of combined cases $(P>0.05)$.

Overall complication rates were infrequent and compare favorably to earlier studies on conventional 
Table 2 Main outcome measure in the two surgery types according to different IOP criteria

\begin{tabular}{|c|c|c|c|}
\hline \multirow{2}{*}{ Success criteria } & \multicolumn{2}{|c|}{ Surgery type } & \multirow{2}{*}{ P-value } \\
\hline & $\begin{array}{c}\text { MSICS-trab } \\
\quad(\mathrm{n}=53)\end{array}$ & $\begin{array}{l}\text { Phaco-trab } \\
(\mathrm{n}=22)\end{array}$ & \\
\hline \multicolumn{4}{|l|}{$\mathrm{IOP} \leq 18 \mathrm{~mm} \mathrm{Hg}$} \\
\hline Without medication (CS) & $33(62.3 \%)$ & $11(50.0 \%)$ & $0.3033^{\mathrm{a}}$ \\
\hline With medication (QS) & $15(28.3 \%)$ & $10(45.5 \%)$ & \\
\hline With or without medication (OS) & $48(90.6 \%)$ & $21(95.4 \%)$ & \\
\hline \multicolumn{4}{|l|}{$I O P \leq 15 \mathrm{~mm} \mathrm{Hg}$} \\
\hline Without medication (CS) & $26(49.0 \%)$ & $9(40.9 \%)$ & $0.5006^{\mathrm{a}}$ \\
\hline With medication (QS) & $11(20.7 \%)$ & $7(31.8 \%)$ & \\
\hline With or without medication (OS) & $37(69.8 \%)$ & $16(72.7 \%)$ & \\
\hline \multicolumn{4}{|l|}{$\mathrm{IOP} \leq 12 \mathrm{~mm} \mathrm{Hg}$} \\
\hline Without medication (CS) & $18(33.9 \%)$ & $7(31.8 \%)$ & $0.9999^{\mathrm{a}}$ \\
\hline With medication (QS) & $9(16.9 \%)$ & $4(18.1 \%)$ & \\
\hline With or without medication (OS) & $27(50.9 \%)$ & $11(50.0 \%)$ & \\
\hline \multicolumn{4}{|l|}{ Target IOP achieved } \\
\hline With or without medication (OS) & $38(71.7 \%)$ & $20(90.9 \%)$ & \\
\hline
\end{tabular}

Abbreviations: CS, complete success; QS, qualified success; OS, overall success.

${ }^{\text {a }}$ As per Chi-square test. trabeculectomy or safe surgery system trabeculectomy. ${ }^{16,20}$ There were four cases of fibrin reaction; two with descemet's detachment and one had hyphema related to scleral tunnel incision used for combined cases.

An important consideration in penetrating glaucoma surgery is the potential for infectious complications as compared with non-penetrating glaucoma surgery. The risk of endophthalmitis with the use of antimetabolite agents is similar between MMC and 5-FU. ${ }^{21,22}$ In our series of combined patients, none of the patients had bleb-related complications or endophthalmitis, although in all of the patients intraoperative MMC was used. In the Collaborative Initial Glaucoma Treatment Study of 285 trabeculectomy patients, the risk of endophthalmitis after 7 years was reported to be $1.1 \%$ $(n=3)$, and there were $14 \%$ patients $(n=40)$ requiring bleb revision. They also found blebitis $(n=8)$, bleb leak $(n=15)$, and hypotony $(n=4)$ in their study cases. ${ }^{23}$ In our study, there were no cases of bleb leak, choroidal detachment, blebitis, or endophthalmitis, although these
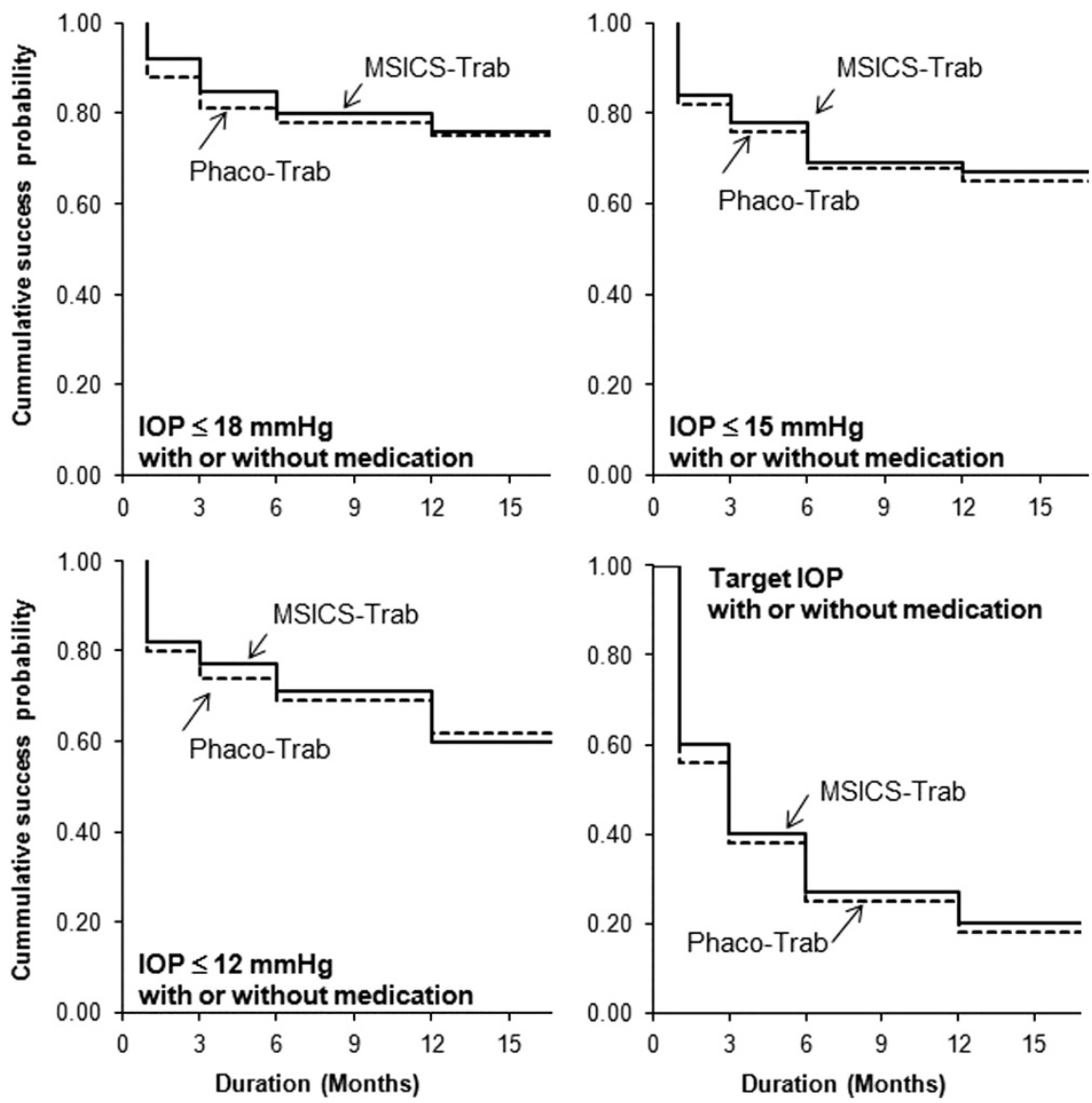

Figure $2 \mathrm{KM}$ plots showing success in the two groups. 
Table 3 Postoperative complications

\begin{tabular}{lcc}
\hline Complications & \multicolumn{2}{c}{ Surgery type } \\
\cline { 2 - 3 } & $\begin{array}{c}\text { MSICS-trab } \\
(\mathrm{n}=53)\end{array}$ & $\begin{array}{c}\text { Phaco-trab } \\
(\mathrm{n}=22)\end{array}$ \\
\hline Fibrin reaction & $3(5.6 \%)$ & $1(4.4 \%)$ \\
Transient hypotony & $3(5.6 \%)$ & $2(9.1 \%)$ \\
Descemet's detachment & $2(3.7 \%)$ & 0 \\
Hyphema & $1(1.8 \%)$ & 0 \\
Secondary glaucoma & $1(1.8 \%)$ & 0 \\
No complications & $44(83.1 \%)$ & $19(86.4 \%)$ \\
\hline
\end{tabular}

complications were reported by earlier studies in trabeculectomy surgery. 3,8,12,22 Postoperative bleb massage was needed in the first week in two cases to control IOP. Three cases had high IOP in spite of antiglaucoma medications after 2 months of surgery; hence, needling revision of the bleb was done to re-establish flow and salvage failing trabeculectomy bleb. There were five cases of transient hypotony without maculopathy in which IOP stabilized without any intervention. There were no complications in $83.1 \%$ of the MSICS-trab and $86.4 \%$ of the phaco-trab group showing statistically insignificant difference. Many recent studies encouraged aggressive postoperative bleb manipulations to form large, diffuse blebs with normal vascularity. ${ }^{16,24}$ In our series, $>90 \%$ blebs were diffuse and mildly elevated with normal vascularity. There were no cases of avascular blebs in any study group.

We adopted the technique of safe surgery system trabeculectomy developed at Moorfields Eye Hospital ${ }^{13-16,25}$ and combined it with cataract surgery (MSICS/phacoemulsification). A fornix-based large conjunctival flap with diffuse and posterior application of mitomycin $C$ resulted in the formation of a diffuse bleb avoiding cystic blebs and 'ring of steel'. To maximize posterior flow, the sides of the scleral tunnel in MSICS/phacoemulsification were left intact. Two adjustable sutures were placed on two sides of the small fistula created by a Kelly's descemet membrane punch that helped in regulating the aqueous flow by good scleral apposition and resistance. ${ }^{25}$ An operating surgeon can perform trabeculectomy through the same scleral tunnel used for MSICS/phacoemulsification after the insertion of PCIOL. The tunnel is then sutured with adjustable sutures, and a watertight conjunctival closure is performed. ${ }^{15}$ This novel type of combined surgery is easy, consumes less time, and has a minimal learning curve. Considering the infrastructure, resources, doubtful follow-up visits, and noncompliance for medical therapy, 'one time' surgical option for managing cataract and glaucoma is the only cost-effective and viable solution. ${ }^{6,20,26-28}$
Sequential surgery has the advantage of greater titratability of IOP but there is increased inconvenience of two surgeries, hence combined surgery is still a gold standard for managing patients of glaucoma with cataract in developing countries. ${ }^{28}$

The shortcomings of this study include its retrospective design and unequal sample size due to preference for economical MSICS over phacoemulsification. There were no cases of bleb-related infection and bleb leak possibly due to small sample size and shorter follow-up period. Prospective, randomized comparative trials with longer follow-up periods, including bleb morphology, are needed to validate this technique. Despite these limitations, the surgical outcomes of this study show that safe surgery system trabeculectomy combined with cataract surgery (MSICS or phacoemulsification) is effective in lowering IOP with minimum complication rates.

\section{Summary}

What was known before

- Safe surgery system trabeculectomy was developed at the Moorfield's Eye Hospital.

- It reduces hypotony and produce diffuse, non-cystic blebs with long-term pressure control.

What this study adds

- Safe surgery system can be effectively combined with any type of cataract surgery (MSICS or phacoemulsification).

- It is a successful solution for advanced glaucoma coexistent with cataract.

\section{Conflict of interest}

The authors declare no conflict of interest.

\section{References}

1 Quigley HA, Broman AT. The number of people with glaucoma worldwide in 2010 and 2020. Br J Ophthalmol 2006; 90: 262-267.

2 Nirmalan PK, Katz J, Robin AL, Krishnadas R, Ramakrishnan R, Thulasiraj RD et al. Utilisation of eye care services in rural south India: the Aravind Comprehensive Eye Survey. Br J Ophthalmol 2004; 88: 1237-1241.

3 Mittal S, Mittal A, Ramakrishnan R. Safety and efficacy of manual small-incision cataract surgery combined with trabeculectomy: comparison with phacotrabeculectomy. Asian J Ophthalmol 2008; 10: 221-229.

4 Bowman RJ, Hay A, Wood ML, Murdoch IE. Combined cataract and trabeculectomy surgery for advanced glaucoma in East Africa; visual and intraocular pressure outcomes. Eye 2010; 24(4): 573-577.

5 Thomas R, Paul P, Muliyil J. Glaucoma in India. J Glaucoma 2003; 12: 81-87.

6 Thomas R, Parikh R, Muliyil J. Comparison between phacoemulsification and the Blumenthal technique of 
manual small-incision cataract surgery combined with trabeculectomy. J Glaucoma 2003; 12: 333-339.

7 Venkatesh R, Sengupta S, Robin A. Mitomycin C augmented trabeculectomy combined with single site manual small incision cataract surgery through a tunnel flap technique. Asia Pac J Ophthalmol 2012; 1: 142-146.

8 Jampel HD, Solus JF, Tracey PA, Gilbert DL, Loyd TL, Jefferys JL et al. Outcomes and bleb-related complications of trabeculectomy. Ophthalmology 2012; 119: 712-722.

9 Friedman DS, Jampel HD, Lubomski LH, Kempen JH, Quigley H, Congdon N et al. Surgical strategies for coexisting glaucoma and cataract. Ophthalmology 2002; 109(10): 1902-1913.

10 Edmunds B, Thompson JR, Salmon JF, Wormald RP. The National Survey of Trabeculectomy. III. Early and late complications. Eye (Lond) 2002; 16: 297-303.

11 Papadopoulos M, Khaw PT. Improving glaucoma filtering surgery. Eye (Lond) 2001; 15: 131-132.

12 Wells AP, Bunce C, Khaw PT. Flap and suture manipulation after trabeculectomy with adjustable sutures: titration of flow and intraocular pressure in guarded filtration surgery. J Glaucoma 2004; 13: 400-406.

13 Dhingra S, Khaw PT. The Moorfields safer surgery system. Middle East Afr J Ophthalmol 2009; 16: 112-115.

14 Khaw PT, Chiang M, Shah P, Sii F, Lockwood A, Khalili A. Enhanced trabeculectomy: the Moorfields safer surgery system. Dev Ophthalmol 2012; 50: 1-28.

15 Stalmans I, Gillis A, Lafaut AS, Zeyen T. Safe trabeculectomy technique: long term outcome. Br J Ophthalmol 2006; 90: 44-47.

16 Gale J, Wells AP. Medium-term outcomes of safe surgery system trabeculectomies. Br J Ophthalmol 2008; 92: 1232-1235.

17 Mario VA. Suggested formula for setting target intraocular pressure. Asian J Ophthalmology 2004; 6: 2-6.

18 Heuer D, Barton K, Grehn F, Shaarawy T, Sherwood M. Consensus on definition of success. In Shaarawy $\mathrm{T}$ (ed) Guidelines on Design and Reporting of Glaucoma Trials. Kugler Publications: Amsterdam, 2009, pp 15-24.

19 Stark WJ, Goyal RK, Awad O, Vito E, Kouzis AC. The safety and efficacy of combined phacoemulsification and trabeculectomy with releasable sutures. Br J Ophthalmol 2006; 90: 146-149.

20 Fiener L, Piltz-Seymour JR. Collaborative Initial Glaucoma Treatment Study: a summary of results to date. Curr Opin Ophthalmol 2003; 14: 106-111.

21 Palanca-Capistrano AM, Hall J, Cantor LB, Morgan L, Hoop J, WuDunn D. Long-term outcomes of intraoperative 5 -fluorouracil versus intraoperative mitomycin $\mathrm{C}$ in primary trabeculectomy surgery. Ophthalmology 2009; 116: 185-190.

22 Bindlish R, Condon GP, Schlosser JD, D'Antonio J, Lauer KB, Lehrer R. Efficacy and safety of mitomycin-C in primary trabeculectomy: five year follow-up. Ophthalmology 2002; 109: 1336-1341.

23 Zahid S, Musch DC, Niziol LM, Lichter PR. Collaborative Initial Glaucoma Treatment Study Group. Risk of endophthalmitis and other long term complications of trabeculectomy in the Collaborative Initial Glaucoma Treatment Study Group. Am J Ophthalmol 2013; 155(4): 674-680.

24 Stead RE, King AJ. Outcome of trabeculectomy with Mitomycin $\mathrm{C}$ in patients with advanced glaucoma. Br J Ophthalmol 2011; 95: 960-965.

25 Jones E, Clarke J, Khaw PT. Recent advances in trabeculectomy technique. Curr Opin Ophthalmol 2005; 16: 107-113.

26 Ramkrishnan R, Khurana M. Surgical management of glaucoma: an Indian perspective. Indian J Ophthalmol 2011; 59(Suppl): 118-122.

27 Congdon NG, Krishnadas R, Friedman DS, Goggins W, Ramakrishnan R, Kader MA et al. A study of initial therapy for glaucoma in southern India: Indian Glaucoma Outcomes and Treatment (INGOT) Study. Ophthalmic Epidemiol 2012; 19(3): 149-158.

28 Thomas R, Sekhar GC, Kumar RS. Glaucoma management in developing countries: medical, laser and surgical options for glaucoma management in countries with limited resources. Curr Opin Ophthalmol 2004; 15: 127-131. 